\title{
DSpace@MIT
}

\author{
MIT Open Access Articles
}

\section{Absolute Continuity of Brownian Bridges Under Certain Gauge Transformations}

The MIT Faculty has made this article openly available. Please share how this access benefits you. Your story matters.

Citation: Nahmod, Andrea R. et al."Absolute Continuity of Brownian Bridges Under Certain Gauge Transformations." Mathematical Research Letters, 18.5 (2011): 875-887.

As Published: http://www.intlpress.com/_newsite/site/pub/pages/journals/items/mrl/content/ vols/0018/0005/00020617/index.php

Publisher: International Press

Persistent URL: http://hdl.handle.net/1721.1/71841

Version: Author's final manuscript: final author's manuscript post peer review, without publisher's formatting or copy editing

Terms of use: Creative Commons Attribution-Noncommercial-Share Alike 3.0 


\title{
ABSOLUTE CONTINUITY OF BROWNIAN BRIDGES UNDER CERTAIN GAUGE TRANSFORMATIONS
}

\author{
ANDREA R. NAHMOD ${ }^{1}$, LUC REY-BELLET ${ }^{2}$, SCOTT SHEFFIELD ${ }^{3}$, AND GIGLIOLA STAFFILANI ${ }^{4}$
}

\begin{abstract}
We prove absolute continuity of Gaussian measures associated to complex Brownian bridges under certain gauge transformations. As an application we prove that the invariant measure for the periodic derivative nonlinear Schrödinger equation obtained by Nahmod, Oh, Rey-Bellet and Staffilani in [20], and with respect to which they proved almost surely global well-posedness, coincides with the weighted Wiener measure constructed by Thomann and Tzvetkov [24]. Thus, in particular we prove the invariance of the measure constructed in [24].
\end{abstract}

\section{INTRODUCTION}

This note is a continuation of the paper [20]. There we constructed an invariant measure for the periodic derivative nonlinear Schrödinger equation (DNLS) (2.1) in one dimension and established global well-posedness, almost surely for data living in its support. This was achieved by introducing a gauge transformation $G$, see (2.12), and by considering the gauged DNLS equation (GDNLS) (2.13) in order to obtain the necessary estimates. We constructed a weighted Wiener measure $\mu$, which we proved to be invariant under the flow of the GDNLS equation, and used it to show the almost surely global well-posedness for the GDNLS initial value problem, in particular almost surely for data in a certain FourierLebesgue space scaling like $H^{\frac{1}{2}-\epsilon}(\mathbb{T})$, for small $\epsilon>0$. To go back to the original DNLS equation we applied the inverse gauge transformation $G^{-1}$ and obtained an invariant measure $\mu \circ G=: \gamma$ with respect to which almost surely global well-posedness is then proved for the DNLS Cauchy initial value problem 5 . On the other hand, Thomann and Tzvetkov [24] constructed a weighted Wiener measure $\nu$ and proposed it as a natural candidate for an invariant measure for the DNLS equation. A natural question, left open in [20], is the absolute continuity of the two measures $\gamma$ and $\nu$ or equivalently, the absolutely continuity of $\mu$ and $\nu \circ G^{-1}$. As shown in this note, this question is easily answered after one understands the absolute continuity between Gaussian measures naturally associated with complex Brownian bridges and their images under certain gauge transformations such as $G$. This is the heart of the matter of this note. At the end we prove that $\mu=\nu \circ G^{-1}$ (or equivalently that $\gamma=\nu$ ) thus in particular establishing the invariance of the measure $\nu$ constructed in [24], see Theorem 2.1. Our results follow by combining the results on

\footnotetext{
${ }^{1}$ The first author is funded in part by NSF DMS 0803160 and a 2009-2010 Radcliffe Institute for Advanced Study Fellowship.

2 The second author is funded in part by NSF DMS 0605058.

${ }^{3}$ The third author is funded in part by NSF CAREER Award DMS 0645585 and a Presidential Early Career Award for Scientists and Engineers (PECASE).

${ }^{4}$ The fourth author is funded in part by NSF DMS 0602678 and a 2009-2010 Radcliffe Institute for Advance Study Fellowship.

${ }^{5}$ In [20] $\mu \circ G$ is called $\nu$; here we relabel it $\gamma$ to a priori distinguish it from the name we give to the one constructed in [24].
} 
global well-posedness and invariant measure for GDNLS (2.13) obtained by Nahmod, Oh, Rey-Bellet and Staffilani in [20] with the explicit computation of the image of the measure under the gauge transformation. The key to understand the latter is to actually understand how the Gaussian part of the measure changes under the gauge since the transformation of the weight is computed easily (see subsection 2.1 below). This is achieved in Theorem 3.1 in Section 3 of this paper.

Certainly there is a vast literature on the topic of Gaussian measures under nonlinear transformations [5, 22, 18, 3, 7, 15, 4] as well as [1] and other references therein. But as we will show below the nature of the gauge transformation $G$ does not fit in the context of these works and a different approach needs to be introduced. For many nonlinear partial differential equations gauge transformations are an essential tool to convert one kind of nonlinearity into another one, where resonant interactions are more manageable and hence estimates can be proved. Therefore we expect the general nature of the central theorem of this note, Theorem 3.1, as well as some of the ideas behind its proof, to be applicable in other situations beyond the DNLS context.

\section{INVARIANCE OF WEIGHTED WIENER MEASURE FOR DNLS}

As stated in the introduction our motivation arises from the recent paper by Nahmod, Oh, Rey-Bellet, and Staffilani [20] we recall now the set up of that paper and formulate the problem that we want to solve here in that context. We consider the derivative nonlinear Schrödinger equation (DNLS) on the circle $\mathbb{T}$, i.e.,

$$
\left\{\begin{array}{l}
u_{t}(x, t)-i u_{x x}(x, t)=\lambda\left(|u|^{2}(x, t) u(x, t)\right)_{x} \\
u(x, 0)=u_{0}(x)
\end{array}\right.
$$

where $x \in \mathbb{T}, t \in \mathbb{R}$ and $\lambda \in \mathbb{R}$ is fixed. In the rest of the paper we will set $\lambda=1$ for simplicity. Our goal is to show that this problem defines a dynamical system, in the sense of ergodic theory. Let us denote by $\Psi(t)$ the flow map associated to our nonlinear equation, i.e., the solution of (2.1), whenever it exists, is given by $u(x, t)=\Psi(t)\left(u_{0}(x)\right)$. Let further $(\mathcal{B}, \mathcal{F}, \nu)$ be a probability space where $\mathcal{B}$ is a space (here $\mathcal{B}$ will be a separable Banach space), $\mathcal{F}$ is a $\sigma$-algebra (here it will always be the Borel $\sigma$-algebra) and $\nu$ is a probability measure. The flow map $\Psi(t)$ define a dynamical system on the probability space $(\mathcal{B}, \mathcal{F}, \nu)$ if

(a) ( $\nu$-almost sure wellposedness.) There exists a subset $\Omega \subset \mathcal{B}$ with $\nu(\Omega)=1$ such that the flow map $\Psi(t): \Omega \rightarrow \Omega$ is well defined and continuous in $t$ for all $t \in \mathbb{R}$.

(b) (Invariance of the measure $\nu$.) The measure $\nu$ is invariant under the flow $\Phi(t)$, i.e.,

$$
\int f(\Psi(t)(u)) d \nu(u)=\int f(u) d \nu(u)
$$

for all $f \in L^{1}(\mathcal{B}, \mathcal{F}, \nu)$ and all $t \in \mathbb{R}$.

The measure $\nu$ here, in a sense, is a substitute for a conserved quantity and in fact $\nu$ is constructed by using a certain conserved quantity. Recall that the DNLS equation (2.1) is 
completely integrable (c.f. [16, 12]) and among the conserved quantities are

$$
\begin{aligned}
\text { Mass: } & m(u) & =\frac{1}{2 \pi} \int|u|^{2} d x, \\
\text { Energy: } & E(u) & =\int\left|u_{x}\right|^{2} d x+\frac{3}{2} \operatorname{Im} \int u^{2} \bar{u} \overline{u_{x}} d x+\frac{1}{2} \int|u|^{6} d x, \\
\text { Hamiltonian: } & H(u) & =\operatorname{Im} \int u \bar{u}_{x} d x+\frac{1}{2} \int|u|^{4} d x .
\end{aligned}
$$

We consider a probability measure $\nu$ which is based on the conserved quantity $E(u)$ (as well as the mass $m(u)$ ). Let us decompose $u=a+i b$ into real and imaginary part, and let us consider first the purely formal but suggestive expression for $\nu$.

$$
d \nu=C^{-1} \chi_{\left\{\|u\|_{L^{2}} \leq B\right\}} e^{-\frac{\beta}{2} N(u)} e^{-\frac{\beta}{2} \int\left(|u|^{2}+\left|u_{x}\right|^{2}\right) d x} \prod_{x \in \mathbb{T}} d a(x) d b(x) .
$$

where

$$
N(u)=\frac{3}{2} \operatorname{Im} \int u^{2} \bar{u} \overline{u_{x}} d x+\frac{1}{2} \int|u|^{6} d x
$$

is the non-quadratic part of the energy $E(u)$. Note that we have added the conserved quantity $\int|u|^{2} d x$ to the quadratic part of $E(u)$ such as to make it positive definite. The constant $\beta>0$ does not play any particular role here and, for simplicity, we choose $\beta=1$. Note however that all the measures for different $\beta$ would be invariant under the flow and they are all mutually singular. The cutoff on the $L^{2}$-norm is necessary to make the measure normalizable since $N(u)$ is not bounded below. We will also see that this measure is welldefined only for $B$ under a certain critical value $B^{*}$.

The expression in (2.5) at this stage is purely formal since there is no Lebesgue measure in infinite dimensions. In order to give a rigorous definition of the measure $\nu$ in (2.5) one needs to:

(a) Make sense of the Gaussian part of the measure (2.5), that is of the formal expression

$$
d \rho=C^{\prime-1} e^{-\frac{1}{2} \int\left(|u|^{2}+\left|u_{x}\right|^{2}\right) d x} \prod_{x \in \mathbb{T}} d a(x) d b(x) .
$$

(b) Construct the measure $\nu$ as a measure absolutely continuous with respect to $\rho$ with Radon-Nikodym derivative

$$
\frac{d \nu}{d \rho}(u)=\mathcal{Z}^{-1} \chi_{\left\{\|u\|_{L^{2}} \leq B\right\}} e^{-\frac{1}{2} N(u)}
$$

i.e., one needs to show that

$$
\mathcal{Z}=\int \chi_{\left\{\|u\|_{L^{2}} \leq B\right\}} e^{-\frac{1}{2} N(u)} d \rho<\infty .
$$

Part (b) goes back to the works of Lebowitz, Rose and Speer [19] and of Bourgain [2] for the term $\int|u|^{6} d x$ part while the integrability of the term involving $\int u^{2} \bar{u} \bar{u}_{x} d x$-and hence the construction of $\nu$ - is proved in [24]; see also Section 5 in [20]. Both terms are critical in the sense that integrability requires that $B$ does not exceed a certain critical value $B^{*}$.

Part (a) is a standard problem in Gaussian measures, treated for example by Kuo [17] and Gross [8]; see also in [20] for details. Indeed the measure $\rho$ can be realized as an honest countably additive Gaussian measure on various Hilbert or Banach spaces depending on 
one's particular needs. For example one can construct $\rho$ as the weak limit of the finitedimensional Gaussian measures

$$
d \rho_{N}=\mathcal{Z}_{0, N}^{-1} \exp \left(-\frac{1}{2} \sum_{|n| \leq N}\left(1+|n|^{2}\right)\left|\widehat{u}_{n}\right|^{2}\right) \prod_{|n| \leq N} d \widehat{a}_{n} d \widehat{b}_{n}
$$

where $\widehat{u}_{n}=\widehat{a}_{n}+i \widehat{b}_{n}$ is the Fourier transform of $u$. For analytical estimates it was convenient in [24] and [20] to consider $\rho$ as measure either on the Hilbert space $H^{\sigma}(\mathbb{T})$ (Sobolev space) for arbitrary $\sigma<1 / 2$ or as a measure on the Banach space $\mathcal{F} L^{s, r}(\mathbb{T}$ ) (FourierLebesgue space [14, 9, 6]) with norm $\|u\|_{\mathcal{F} L^{s, r}(\mathbb{T})}:=\left\|\langle n\rangle^{s} \widehat{u}\right\|_{\ell_{n}^{r}(\mathbb{Z})}$ and with the conditions $2 \leq r<\infty$ and $(s-1) r<-1$. Note $\mathcal{F} L^{s, r}(\mathbb{T})$ scales like $H^{\sigma}(\mathbb{T})$ where $\sigma=s+\frac{1}{r}-\frac{1}{2}$ and the condition $(s-1) r<-1$ is equivalent to $\sigma<1 / 2$.

From a probabilistic stand point, however, and to connect the measure $\rho$ with the results in subsequent sections, it is also natural to realize this measure on the space of complexvalued $2 \pi$-periodic continuous functions $C(\mathbb{T}, \mathbb{C})$. The measure $\rho$ is closely related to the (complex) Brownian bridges $Z_{u_{o}}(x)$ where $0 \leq x \leq 2 \pi$ and $Z_{u_{o}}(0)=Z_{u_{o}}(2 \pi)=u_{o}$. Indeed let $\rho\left(\cdot \mid u_{o}\right)$ denote the measure $\rho$ conditioned on the event $\left\{u(0)=u(2 \pi)=u_{o}\right\}$. If $\kappa$ denotes the distribution of $u_{o}$, then $\kappa$ is a complex Gaussian probability measure and we have $\rho(\cdot)=\int_{\mathbb{C}} \rho\left(\cdot \mid u_{o}\right) d \kappa\left(u_{o}\right)$. Then $\rho\left(\cdot \mid u_{o}\right)$ is absolutely continuous with respect to the probability distribution $P_{u_{o}}$ of the complex Brownian bridge with

$$
\frac{d \rho\left(\cdot \mid u_{o}\right)}{d P_{u_{o}}}=\mathcal{Z}_{u_{o}}^{-1} e^{-\frac{1}{2} \int_{0}^{2 \pi}|u|^{2} d x} .
$$

This can be easily seen for example by considering the finite-dimensional distribution of $\rho$.

By combining the results obtained by Nahmod, Oh, Rey-Bellet, and Stafillani in [20] for the gauged equation and the results in the present paper we will prove the following theorem:

Theorem 2.1. The DNLS equation (2.1) is $\nu$-almost surely well-posed and the measure $\nu$ is invariant for the flow map $\Psi(t)$ for (2.1).

We now explain why in order to prove Theorem 2.1 one needs to introduce a gauge transformation. We go back to the existence of (local) solutions to (2.1). By examining the equation one sees there is a derivative loss arising from the nonlinear term $\left(|u|^{2} u\right)_{x}=$ $u^{2} \bar{u}_{x}+2|u|^{2} u_{x}$ and hence for low regularity data one must somehow make up for this loss. Since the worse resonant interactions occur on the second term $|u|^{2} u_{x}$ a key idea is to suitably gauge transform the equation to get rid of it, see [11, 12, 23, 13, 10]. In the periodic context a suitable gauge transformation was introduced by Herr [13]. For $f \in L^{2}(\mathbb{T})$ let us define

$$
G(f)=e^{-i J(f)} f, \quad \text { with } \quad J(f)(x)=\frac{1}{2 \pi} \int_{0}^{2 \pi} \int_{\theta}^{x}\left(|f(y)|^{2}-m(f)\right) d y d \theta,
$$

and note that the inverse of $G$ is simply given by $G^{-1}(f)=e^{i J(f)} f$. Under the gauge $G$, if $u$ is a solution of the DNLS equation (2.1) then $w(x, t)=G(u(x, t))$ is a solution to what we call the GDNLS equation

$$
w_{t}-i w_{x x}-2 m(w) w_{x}=-w^{2} \bar{w}_{x}+\frac{i}{2}|w|^{4} w-i \psi(w) w-i m(w)|w|^{2} w
$$


where

$$
\psi(w):=-\frac{1}{\pi} \int_{\mathbb{T}} \operatorname{Im}\left(w \bar{w}_{x}\right) d x+\frac{1}{4 \pi} \int_{\mathbb{T}}|w|^{4} d x-m(w)^{2} .
$$

The main result of the present paper is to show how the measure $\nu$ is transformed under the gauge transformation $G$. The image of $\nu$ under $G$ is denoted 6 by $\mu$ and is, by definition, given by

$$
\mu(A):=\nu\left(G^{-1}(A)\right)=\nu(\{x ; G(x) \in A\}),
$$

where $A$ is any measurable set. We will use the notation $\mu=\nu \circ G^{-1}$ in the sequel. We have

Theorem 2.2. For sufficiently small $B$, the measure $\mu=\nu \circ G^{-1}$ is absolutely continuous with respect to the Gaussian measure $\rho$ and we have

$$
\frac{d \mu}{d \rho}(w)=\tilde{\mathcal{Z}}^{-1} \chi_{\left\{\|w\|_{L^{2}} \leq B\right\}} e^{-\frac{1}{2} \mathcal{N}(w)},
$$

where

$$
\mathcal{N}(w)=-\frac{1}{2} \operatorname{Im} \int w^{2} \overline{w w_{x}} d x+2 m(w) \operatorname{Im} \int w \overline{w_{x}} d x-\frac{1}{2} m(w) \int|w|^{4} d x+2 \pi m(w)^{3},
$$

and $\tilde{\mathcal{Z}}$ is a normalization constant.

For the measure $\mu$, as given by (2.15), the following result is proved in [20], see Theorem $6.3,6.5,7.1$, and 7.2 .

Theorem 2.3. The GDNLS equation (2.13) is $\mu$-almost surely well-posed and the measure $\mu$ is invariant for the flow map $\Phi(t)$ for (2.13).

Remark 2.4. In [13] and [20] one actually performs another supplementary transformation to get rid of the term $2 m(w) w_{x}$ on the left hand side of (2.13). Indeed if we set $v(x, t)=$ $w(x-2 \operatorname{tm}(w), t)$ then $v$ is a solution of

$$
v_{t}-i v_{x x}=-v^{2} \bar{v}_{x}+\frac{i}{2}|v|^{4} v-i \psi(v) v-i m(v)|v|^{2} v .
$$

A simple argument given in section 7 of [20] show that the measure $\mu$ is invariant for both the flow maps for (2.13) and (2.16).

To conclude one notes that Theorem 2.1 follows immediately from Theorem 2.2 and 2.3 . We are thus left to prove Theorem 2.2 .

2.1. An heuristic introduction of $\mu$. To understand the form of the measure $\mu$ we give here a purely heuristic argument, a rigorous proof is given in the next section. Let us first recall how the invariants for DNLS transform under $G$. Since $u=e^{i J(w)} w$ we have $m(u)=m(w)$ and $u_{x}=e^{i J(w)}\left(w_{x}+i J(w)_{x} w\right)$ with $J(w)_{x}=|w|^{2}-m(w)$ and we obtain after straightforward computations

$$
\begin{aligned}
H(u) & =\operatorname{Im} \int_{\mathbb{T}} u \bar{u}_{x} d x+\frac{1}{2} \int_{\mathbb{T}}|u|^{4} d x \\
& =\operatorname{Im} \int_{\mathbb{T}} w \bar{w}_{x}-\frac{1}{2} \int_{\mathbb{T}}|w|^{4} d x+2 \pi m(w)^{2}=: \mathscr{H}(w),
\end{aligned}
$$

\footnotetext{
${ }^{6}$ This $\mu$ is not yet the same as the $\mu$ constructed in [20] that we referred to in the Introduction. But it will indeed be the same as a consequence of 2.15 after we prove Theorem 2.2
} 
and

$$
\begin{aligned}
u_{x} \overline{u_{x}} & =\left(w_{x}+i J(w)_{x} w\right)\left(\overline{w_{x}}-i J(w)_{x} \bar{w}\right) \\
& =w_{x} \overline{w_{x}}-2 \operatorname{Im} w^{2} \overline{w w_{x}}+2 m(w) \operatorname{Im} w \overline{w_{x}}+\left(|w|^{6}-2 m|w|^{4}+m(w)^{2}|w|^{2}\right),
\end{aligned}
$$

as well as

$$
u^{2} \overline{u u_{x}}=w^{2} \overline{w w_{x}}-i|w|^{6}+i m(w)|w|^{4} .
$$

Hence by using (2.3), (2.18), (2.19) we find

$$
\begin{aligned}
E(u)= & \int w_{x} \overline{w_{x}} d x-\frac{1}{2} \operatorname{Im} \int w^{2} \overline{w w_{x}} d x+2 m(w) \operatorname{Im} \int w \overline{w_{x}} d x \\
& -\frac{1}{2} m(w) \int|w|^{4} d x+2 \pi m(w)^{3} \\
=: & \mathcal{E}(w) .
\end{aligned}
$$

Remark 2.5. Notice that $\mathcal{E}(w)$ involves the other conserved quantities $\mathscr{H}(w)$ and $m(w)$ and if we define

$$
\mathscr{E}(w):=\int_{\mathbb{T}}\left|w_{x}\right|^{2} d x-\frac{1}{2} \operatorname{Im} \int_{\mathbb{T}} w^{2} \bar{w} \overline{w_{x}} d x+\frac{1}{4 \pi}\left(\int_{\mathbb{T}}|w(t)|^{2} d x\right)\left(\int_{\mathbb{T}}|w(t)|^{4} d x\right),
$$

we then have

$$
E(u)=\mathscr{E}(w)+2 m \mathscr{H}(w)-2 \pi m^{3}=\mathcal{E}(w),
$$

and so $\mathscr{E}(w)$ is also a conserved quantity. One could build invariant measures using $\mathscr{E}(w)$ rather than $\mathcal{E}(w)$ but they would turn out to be equivalent measures.

Let us pretend that the measure $\nu$ is the measure with density

$$
\chi_{\left\{\|u\|_{L^{2}} \leq B\right\}} e^{-\frac{1}{2} N(u)} e^{-\frac{1}{2} \int\left(|u|^{2}+\left|u_{x}\right|^{2}\right) d x}=\chi_{\left\{\|u\|_{L^{2}} \leq B\right\}} e^{-\frac{1}{2} E(u)-\frac{1}{2} \int|u|^{2} d x}
$$

with respect to the (nonexistent!) infinite dimensional Lebesgue measure $\prod_{x \in \mathbb{T}} d a(x) d b(x)$. Let us assume furthermore that this nonexistent Lebesgue measure is left invariant under $G$. Then we would simply obtain from (2.20) that

$$
\begin{aligned}
d \mu & =\tilde{C}^{-1} \chi_{\left\{\|w\|_{L^{2}} \leq B\right\}} e^{-\frac{1}{2} \mathcal{E}(w)-\frac{1}{2} \int|w|^{2} d x} \prod_{x \in \mathbb{T}} d a(x) d b(x) \\
& =\mathcal{Z}^{-1} \chi_{\left\{\|w\|_{L^{2}} \leq B\right\}} e^{-\frac{1}{2} \mathcal{N}(w)} d \rho,
\end{aligned}
$$

where

$$
\mathcal{N}(w)=-\frac{1}{2} \operatorname{Im} \int w^{2} \overline{w w_{x}} d x+2 m(w) \operatorname{Im} \int w \overline{w_{x}} d x-\frac{1}{2} m(w) \int|w|^{4} d x+2 \pi m(w)^{3}
$$

is the nonquadratic part of the energy $\mathcal{E}(w)$.

The crucial problem to understand rigorously the transformation of $\mu$ under $G$ is actually to understand the transformation of the Gaussian part $\rho$ of $\mu$ under $G$ since the transformation of the weight is computed easily as in the formal computation above. This is achieved in Theorem 3.1 below where in order to analyze the transformation of $\rho$ the main ingredients will be:

(i) The relation between $\rho$ and Brownian bridges, see eq. (2.11).

(ii) The well-known fact that a Brownian bridge can be obtained by conditioning a Brownian motion to return at its starting point. 
(iii) The conformal invariance of Brownian motion. Note that since $w=e^{-i J(u)} u, J(u)=$ $J(w)$ and $J(u)=J(|u|)$, it is more convenient to consider this transformation in terms of the variables

$$
|w|=|u|, \quad \arg (w)=\arg (u)-i J(|u|) .
$$

By conformal invariance of Brownian motion and (ii), $|u|$ and $\arg (u)$ have a Gaussian distribution after a suitable reparametrization. The transformation (2.26) is easy to understand. In particular if we condition on $|u|$, the transformation of $\arg (u)$ is a simple translation by a fixed vector which leads to the next item (iv).

(iv) The Cameron-Martin formula for the transformation of Gaussian measure under a translation by a fixed vector, see e.g. [5, 1]. We will use here the following special case of the Cameron-Martin theorem.

Theorem 2.6. (Cameron-Martin theorem for real Brownian bridge). Let $X(s), 0 \leq s \leq S$, be a real Brownian bridge, with $X(0)=X(S)=x_{o}$ and law $R_{x_{o}}$. Let $k(s), 0 \leq s \leq S$, be an absolutely continuous real-valued function such that $k(0)=k(S)=k_{o}$ and $\int_{0}^{S}\left|k^{\prime}(s)\right|^{2} d s<\infty$. Then the law of $X(s)+k(s)$ is absolutely continuous with respect to $R_{x_{o}+k_{o}}$ with Radon-Nikodym derivative

$$
\exp \left(\int_{0}^{S} k^{\prime}(s) d X(s)-\frac{1}{2} \int_{0}^{S}\left|k^{\prime}(s)\right|^{2} d s\right) .
$$

\section{BROWNIAN BRIDGES UNDER GAUGE TRANSFORMATIONS}

Let $Z_{u_{o}}(x)$ be a standard complex Brownian bridge 7 on the interval $0 \leq x \leq 2 \pi$ and with $Z_{u_{o}}(0)=Z_{u_{o}}(2 \pi)=u_{o}$. The law of $Z_{u_{o}}$ is denoted by $P_{u_{o}}$ and is a Gaussian probability on $\left\{Z \in C(\mathbb{T} ; \mathbb{C}), Z(0)=u_{o}\right\}$. Since no confusion arises we will omit the index $u_{o}$ in the sequel and denote the Brownian bridge simply by $Z$ and its probability distribution by $P$. We consider first the transformation of a complex Brownian bridge under a class of transformations which contains in particular the gauge transformation $G$ given in (2.12).

We assume that the map $G$ satisfies the following condition

(C) The map $G: C(\mathbb{T}, \mathbb{C}) \rightarrow C(\mathbb{T}, \mathbb{C})$ has the form

$$
G(Z)(x)=e^{-i J(Z)(x)} Z(x),
$$

where $J: C(\mathbb{T}, \mathbb{C}) \rightarrow C(\mathbb{T}, \mathbb{R})$ depends only on $|Z|$ and is such that

$$
\frac{d}{d x} J(Z)(x)=h(|Z|)(x),
$$

and $h(|Z|)(x)$ is continuous in $x$ for $P$-almost all choices of the process $|Z|$.

The gauge transformation (2.12) in Section 1 satisfies condition (C) since we have

$$
\begin{aligned}
J(Z)(x) & =\frac{1}{2 \pi} \int_{0}^{2 \pi} \int_{\theta}^{x}\left(|Z(y)|^{2}-\frac{1}{2 \pi} \int_{0}^{2 \pi}|Z(\xi)|^{2} d \xi\right) d y d \theta, \\
h(|Z|)(x) & =|Z(x)|^{2}-\frac{1}{2 \pi} \int_{0}^{2 \pi}|Z(\xi)|^{2} d \xi .
\end{aligned}
$$

\footnotetext{
${ }^{7}$ Although in the probability literature it is customary to use $t$ as the variable of Brownian motions, here we use $x$ instead in order not to confuse it with the time $t$ of the evolution equation [2.1).
} 
Theorem 3.1. Let $Z$ be a standard complex Brownian bridge with law P. Let $G$ be a map which satisfies the condition $(\mathbf{C})$. If we have

$$
\mathbb{E}_{P}\left[\exp \left(\operatorname{Im} \int_{0}^{2 \pi} h(|Z|)(x) Z(x) d \bar{Z}(x)-\frac{1}{2} \int_{0}^{2 \pi}|h(|Z|)(x)|^{2}|Z(x)|^{2} d x\right)\right]=1,
$$

then $P \circ G^{-1}$ is absolutely continuous with respect to the Brownian bridge P with Radon-Nikodym derivative

$$
\exp \left(\operatorname{Im} \int_{0}^{2 \pi} h(|Z|)(x) Z(x) d \bar{Z}(x)-\frac{1}{2} \int_{0}^{2 \pi}|h(|Z|)(x)|^{2}|Z(x)|^{2} d x\right) .
$$

Remark 3.2. Assume for a moment that we are not in a periodic setting, that $Z$ is a standard Brownian motion instead of a Brownian bridge, and that

$$
J(Z)(x)=\int_{0}^{x} f(|Z|(t)) d t
$$

for some real-valued continuous function $f$. The expression in the right hand side of (3.7) looks very similar to the corresponding one in (3.2), but it is actually easier to handle since it is non anticipative, in the sense that it depends on the Brownian motion up to "time" $x$ and not later. Thanks to this fact the Radon-Nikodym derivative for the transformation $G$ can be computed as a consequence of Girsanov formula (e.g. [21]). Indeed if we set $R=J(Z)$ then we have

$$
\tilde{Z} \equiv G(Z)=e^{-i R} Z
$$

By Ito's formula we have $d R=f(|Z|) d x$ and

$$
\begin{aligned}
d \tilde{Z} & =-i e^{-i R} Z d R+e^{-i R} d Z-\frac{1}{2} e^{-i R} Z d R^{2}+i e^{-i R} d R d Z+0 \frac{1}{2} d Z^{2} \\
& =e^{-i J(Z)}(-i Z) f(|Z|) d x+e^{-i J(Z)} d Z \\
& =-i \tilde{Z} f(|\tilde{Z}|) d x+e^{-i J(Z)} d Z
\end{aligned}
$$

where we have used that $|\tilde{Z}|=|Z|$. Since $J(Z)(x)$ is a nonanticipating functional $e^{-i J(Z)} d Z$ is a Brownian motion (see [21]) and therefore $\tilde{Z}$ has the same law as the solution of the SDE

$$
d \tilde{Z}=-i \tilde{Z} f(\tilde{Z}) d x+d Z .
$$

An application of Girsanov Theorem gives now the form of the Radon-Nikodym derivative as in (3.6).

Proof of Theorem 3.1 The remark above explains why the kind of gauge transformations we consider cannot be studied directly by the Girsanov Theorem and some manipulation needs to be performed.

In the course of the proof we will use some properties of the complex Brownian motion which we denote by $B(x)$ (again we omit from the notation the choice of $B(0)$.) We recall first the well-known fact, see e.g. [21], that a Brownian bridge $Z(x)$ is obtained from a Brownian motion by conditioning $B$ on the event $\{B(2 \pi)=B(0)\}$.

Furthermore we will use the conformal invariance of Brownian motion, that is if $A=$ $A_{1}+i A_{2}$ is a complex Brownian motion, and $\phi$ is analytic function then $B=\phi(A)$ is, after a suitable time change, again a complex Brownian motion (see e.g. [21], Example 8.22). For $B(x)=\exp (A(s))$ the time change is given by

$$
x=x(s)=\int_{0}^{s}\left|e^{A(r)}\right|^{2} d r=\int_{0}^{s} e^{2 A_{1}(r)} d r \quad \frac{d x}{d s}=\left|e^{A(s)}\right|^{2}=|B(x(s))|^{2},
$$


or equivalently

$$
s(x)=\int_{0}^{x} \frac{d r}{|B(r)|^{2}}, \quad \frac{d s}{d x}=\frac{1}{|B(x)|^{2}} .
$$

If we write $B(x)$ in polar coordinate

$$
B(x)=|B(x)| e^{i \arg (B)(x)},
$$

we have

$$
A(s)=A_{1}(s)+i A_{2}(s)=\log |B(x(s))|+i \arg (B)(x(s)),
$$

and $A_{1}$ and $A_{2}$ are real independent Brownian motions.

In view of conditioning the Brownian motion to obtain a Brownian bridge we are interested in $B(x)$ for $0 \leq x \leq 2 \pi$ and thus we introduce the stopping time

$$
S=\inf \left\{s ; \int_{0}^{s}\left|e^{A(r)}\right|^{2} d r=2 \pi\right\},
$$

and remark, for future use, the important fact that the stopping time $S$ depends only on the real part $A_{1}(s)$ of $A(s)$, or equivalently only $|B|(x)$.

For the Brownian bridge $Z(x)$ let us define $W(s)=W_{1}(s)+i W_{2}(s)$ where

$$
W_{1}(s)=\log |Z(x(s))|, \quad W_{2}(s)=\arg (Z)(x(s)),
$$

where $x(s)=\int_{0}^{s} e^{2 W_{1}(r)} d r$ and $0 \leq x \leq 2 \pi$ is equivalent to $0 \leq s \leq S$. Furthermore we denote by $Q$ the law of $W$. The stopping time depends only $|Z|$, so once we condition on the process $W_{1}(s)=\log |Z(x(s))|$, the conditional law of the process $W_{2}(s)=\arg (Z)(x(s))$ is now the law of a real Brownian bridge on the interval $0 \leq s \leq S$.

If we define $\widetilde{W}=\mathcal{L}(W)=\widetilde{W}_{1}+i \widetilde{W}_{2}$ by

$$
\begin{aligned}
\mathcal{L}(W)(s) & :=W_{1}(s)+i\left[W_{2}(s)-J(|Z|)(x(s))\right] \\
& =W_{1}(s)+i\left[W_{2}(s)-J\left(e^{W_{1}}\right)(s)\right],
\end{aligned}
$$

then we have

$$
e^{\mathcal{L}(W)(s)}=G(Z)(x(s)),
$$

where $G$ is as in (3.1). From (3.14) we observe first that the (marginal) law of $W_{1}$, under $Q$, and the (marginal) law of the real part $\widetilde{W}_{1}$ of $\mathcal{L}(W)$, under $Q \circ \mathcal{L}^{-1}$, coincide. Furthermore the conditional law of $W_{2}$, conditioned on the real part $W_{1}$, is the law of a real Brownian bridge on the interval $[0, S]$. Therefore from (3.14) the conditional law of $\widetilde{W}_{2}$, conditioned on the real part $W_{1}=\widetilde{W}_{1}$, can be computed using Cameron-Martin formula, Theorem 2.6, since $\widetilde{W}_{2}$ is obtained from $W_{2}$ by translating by a function which depends only on $W_{1}$.

Since

$$
\frac{d J(u(x(s)))}{d s}=(J(u))^{\prime}(x(s)) \frac{d x(s)}{d s}=h(|u|)(x(s)) \frac{d x(s)}{d s},
$$

the Cameron-Martin formula implies that the Radon-Nikodym derivative of the law of imaginary part of $\mathcal{L}(W)$ with respect to the law of a real brownian bridge on the interval $0 \leq s \leq S$ is given by

$$
\exp \left(\int_{0}^{S} h(|Z|)(x(s)) \frac{d x}{d s} d W_{2}(s)-\frac{1}{2} \int_{0}^{S} h(|Z|)^{2}(x(s))\left(\frac{d x}{d s}\right)^{2} d s\right) .
$$


To conclude we finally re-express the Radon-Nikodym derivative in terms of $Z$ and $x$. We have

$$
d W(s)=\frac{1}{Z(x(s))} \frac{d x}{d s} d Z(x(s))=\bar{Z}(x(s)) d Z(x(s))
$$

and thus

$$
\int_{0}^{S} h(|Z|)(x(s)) \frac{d x}{d s} d W_{2}(s)=\operatorname{Im} \int_{0}^{2 \pi} h(|Z|)(x) \bar{Z} d Z(x)
$$

and

$$
\int_{0}^{S} h(|Z|)^{2}(x(s))\left(\frac{d x}{d s}\right)^{2} d s=\int_{0}^{2 \pi}|h(|Z|)(x)|^{2}|Z(x)|^{2} d x .
$$

This concludes the proof of Theorem 3.1

3.1. Application to the periodic derivative NLS. Let us consider the measure $\mu$ given in the introduction, see (2.7) and (2.8). In this section we prove Theorem 2.2 using Theorem 3.1 .

Proof of Theorem 2.2 We note that, using (2.19), the Radon-Nykodym derivative $\frac{d \nu}{d \rho}$ transforms under the gauge $G$ as

$$
N\left(G^{-1}(w)\right)=\frac{3}{2} \operatorname{Im} \int w^{2} \overline{w w_{x}} d x-\int|w|^{6} d x+\frac{3}{2} m \int|w|^{4} d x
$$

Furthermore by the results of [2, 24, 20] $\chi_{\left\{\|w\|_{L^{2}} \leq B\right\}} e^{-\frac{1}{2} N\left(G^{-1}(w)\right)} \in L^{1}(\rho)$ for sufficiently small $B$. Therefore it is enough to consider how $\rho$ transforms under the gauge transformation, i.e., we consider the measure $\tilde{\rho}=\rho \circ G^{-1}$. Without cutoff on $\|u\|_{L^{2}}$ one cannot expect $\tilde{\rho}$ to be absolutely continuous with respect to $\rho$, but all we really need is that the restriction of $\tilde{\rho}$ on $\left\{m(w) \leq \frac{B^{2}}{2 \pi}\right\}$ be absolutely continuous with respect to $\rho$. Alternatively we can incorporate the cutoff in $J$ by redefining $J$ to be

$$
J(w)(x):= \begin{cases}\frac{1}{2 \pi} \int_{0}^{2 \pi} \int_{\theta}^{x}\left(|w(y)|^{2}-m(w)\right) d y d \theta & \text { if } m(w) \leq \frac{B^{2}}{2 \pi} \\ 0 & \text { otherwise. }\end{cases}
$$

so that we have

$$
\frac{d}{d x} J(w)(x)=h(|w|)(x)= \begin{cases}|w(x)|^{2}-m(w) & \text { if } m(w) \leq \frac{B^{2}}{2 \pi} \\ 0 & \text { otherwise. }\end{cases}
$$

By the results in [2, 24, 20]

$$
\begin{aligned}
& \exp \left(\operatorname{Im} \int h(|w|) w \bar{w}_{x} d x-\frac{1}{2} \int h(|w|)^{2}|w|^{2} d x\right)= \\
& \chi_{\left\{m(w) \leq \frac{B^{2}}{2 \pi}\right\}} \exp \left(\operatorname{Im} \int\left(|w|^{2}-m(w)\right) w \bar{w}_{x} d x-\frac{1}{2} \int\left(|w(x)|^{2}-m(w)\right)^{2}|w(x)|^{2} d x\right)
\end{aligned}
$$

belongs to $L^{1}(\rho)$ for sufficiently small $B$. By conditioning on $\left\{u(0)=u(2 \pi)=u_{o}\right\}$ and using equation (2.11) we conclude that the Novikov condition (3.5) is satisfied for almost every $u_{o}$. Therefore using Theorem 3.1 the Radon-Nikodym derivative $\frac{d \tilde{\rho}}{d \rho}$ is given by (3.22). Finally combining the equations (3.22) and (3.19) we obtain equation (2.15). 
Acknowledgment. Andrea R. Nahmod and Gigliola Staffilani would like to warmly thank the Radcliffe Institute for Advanced Study at Harvard University for its wonderful hospitality while part of this work was being carried out. They also thank their fellow Fellows for the stimulating environment they created.

\section{REFERENCES}

[1] Bogachev, V.I. Gaussian measures, Mathematical Surveys and Monographs, 62 American Mathematical Society, Providence, RI, [1998]

[2] Bourgain, J., Periodic nonlinear Schrödinger equation and invariant measures, Commun. Math. Phys. 166 pp. 1-26 [1994].

[3] Buckdahn, R. Anticipative Girsanov transformations, Probab. Th. Rel. Fields 89, pp. 211-238 [1991].

[4] Cambronero, S. H. McKean, H, The Ground State Eigenvalue of Hills Equation with White Noise Potential, Communications on Pure and Applied Mathematics LII, pp. 1277-1294, [1999].

[5] Cameron, R. H. and Martin, W. T. Transformation of Wiener integrals under translations, Annals of Math. 45 no. 2, pp. 386-396 [1944].

[6] Christ, M, Power series solution of a nonlinear Schrödinger equation, Mathematical Aspects of Nonlinear Dispersive Equations, Ann. of Math. Stud. 163, Princeton University Press, Princeton, NJ, pp. 131-155 [2007].

[7] Enchev, O. Nonlinear Transformations on the Wiener Space, The Annals of Probability, 21, no 4, pp. 2169-2188 [1993].

[8] Gross, L., Abstract Wiener spaces, Proc. 5th Berkeley Sym. Math. Stat. Prob. 2, pp. 31-42, [1965].

[9] Grünrock, A., Bi and Trilinear Schrödinger estimates in one space dimension with applications to cubic NLS and DNLS, IMRN 41, pp. 2525-2558 [2005].

[10] Grünrock, A. and Herr, S., Low Regularity local well-posedness of the derivative nonlinear Schrödinger equation with periodic data, SIAM J. Math. Anal. 39, no. 6 pp. 1890-1920, [2008].

[11] Hayashi, N. and Ozawa, T., On the derivative nonlinear Schrödinger equation, Physica D 55 no. 1-2, pp. 14-36 [1992].

[12] Hayashi, N. and Ozawa, T. Finite energy solutions of nonlinear Schrödinger equation of derivative type, SIAM J. of Math. Anal. 25 no. 6, pp. 1488-1503 [1994].

[13] Herr, S. On the Cauchy problem for the derivative nonlinear Schrödinger equation with periodic boundary condition, IMRN, Article ID 96763, pp. 1-33, [2006].

[14] Hörmander, L., The Analysis of Linear Partial Differential Operators. II, Grundlehren Math. Wiss. 257, Springer-Verlag, Berlin, [1983].

[15] Kallianpur G. and Karandikar, R. L., Nonlinear Transformations of the Canonical Gauss Measure on Hilbert Space and Absolute Continuity, Acta Applicandae Mathematicae 35, pp 63-102, [1994].

[16] Kaup, D.J. and Newell, A.C. An exact solution for the derivative nonlinear Schrödinger equation, J. of Math. Physics 19 no. 4, pp. 798-801 [1978].

[17] Kuo, H., Gaussian Measures in Banach Spaces, Lec. Notes in Math. 463, Springer-Verlag, New York, [1975].

[18] Kusuoka, S. The nonlinear transformation of Gaussian measure on Banach space and its absolute continuity (I), J. Fac. Sci. Univ. Tokyo Sect. IA Math. 29, no. 3, pp. 567-597 [1982].

[19] Lebowitz, J., Rose, H., and Speer, E., Statistical mechanics of the nonlinear Schrödinger equation J. Stat. Phys. 50 no. 3-4, pp. 657-687 [1988].

[20] Nahmod, A. R., Oh, T., Rey-Bellet, L. and Staffilani, G., Invariant weighted Wiener measures and almost sure global well-posedness for the periodic derivative NLS. JEMS, to appear.

[21] Oksendal, B. Stochastic Differential Equations, Springer-Verlag, Fifth Edition, [1998].

[22] Ramer, R. On nonlinear tranformations of Gaussian measures, J. Functional Anal. 15, pp. 166-187 [1974].

[23] Takaoka, H., Well-posedness for the one dimensional nonlinear Schrödinger equation with the derivative nonlinearity, Adv. Differential Equations, 4 , no.4 pp. 561-580 [1999].

[24] Thomann, L. and Tzvetkov, N., Gibbs measure for the periodic derivative nonlinear Schrödinger equation, Nonlinearity 23 pp. 2771-2791 [2010]. 
1 Department of Mathematics, University of Massachusetts, 710 N. Pleasant Street, AMHERST MA 01003

E-mail address: nahmodemath.umass.edu

2 Department of Mathematics, University of Massachusetts, 710 N. Pleasant Street, AMHERST MA 01003

E-mail address: lucemath.umass.edu

${ }^{3}$ Department of Mathematics, Massachusetts Institute of Technology, 77 Massachusetts AVEnUE, CAMBRIDGE, MA 02139

E-mail address: sheffield@math.mit.edu

${ }^{4}$ Department of Mathematics, Massachusetts Institute of Technology, 77 Massachusetts AVEnUE, CAMBRIDGE, MA 02139

E-mail address: gigliola@math.mit.edu 